\title{
Contribution à l'étude des plantes médicinales utilisées dans le traitement des abcès dans le territoire de Bikoro, province de l'Equateur en RDC
}

\section{Guy ILUMBE BAYELI ${ }^{1 *}$, Véronique JOIRIS ${ }^{2}$, Gustave Nyamangombe LOHANDJOLA $^{1}$ et Jean-pierre HABARI ${ }^{3}$}

${ }^{1}$ Laboratoire d'Ethnobiologie, Département de Biologie, Faculté des Sciences, Université de Kinshasa, B.P 190 Kinshasa XI, RD Congo.

${ }^{2}$ Faculté de Philosophie et Sc. Sociales, Université Libre de Bruxelles, Campus du Solbosch CP124, avenue F.D. Roosevelt 50, 1050 Bruxelles.

${ }^{3}$ Herbarium de Kinshasa (IUK), Département de Biologie, Faculté des Sciences, Université de Kinshasa, B.P 190 Kinshasa XI, RD Congo.

*Auteur correspondant ; E-mail: guy_ilumbe@yahoo.com

\section{RESUME}

La présente étude contribue à l'inventaire des plantes médicinales et des recettes médicamenteuses utilisées dans les traitements des abcès dans le territoire de Bikoro, province de l'Equateur en République Démocratique du Congo. Nommé sous l'appellation de «Matuku» par les populations locales, les abcès constituent une des affections les plus rencontrées dans le territoire de Bikoro. L'absence de structures sanitaires adéquates voir même inexistantes fait que les populations de Bikoro ont recours à la médecine traditionnelle pour se soigner. C'est ce qui a motivé la réalisation de la présente étude.L'entretien semi-direct structuré sur un questionnaire au cours duquel 32 tradipraticiens, ayant au moins 10 ans d'expérience, a permis de recenser au total 43 espèces médicinales reparties en 39 genres et 27 familles dont les mieux représentées étaient les Euphorbiaceae (3 genres et 4 espèces), les Asteraceae (3/3), les Commelinaceae (2/2) et les Araceae $(2 / 2)$. Isolées ou en combinaison, ces plantes entrent dans la formulation de 50 recettes médicamenteuses. Les feuilles et les écorces du tronc-tige constituent les principaux organes sollicités. Préférentiellement, la préparation des remèdes se fait par trituration-pilage et leur administration par application locale c'est-à-dire directement sur l'abcès. En rapport avec leur importance culturelle, les espèces: Boerhavia diffusa L., Bridelia micrantha (Hoshst.) Baill.et Psydrax palma (K. Schum.) Bridson sont les mieux indiquées.

(C) 2019 International Formulae Group. All rights reserved.

Mots clés : Plantes médicinales, Ethnobotanique, abcès, République Démocratique du Congo.

\section{Contribution to the study of medicinal plants used in the treatment of abscesses in the territory of Bikoro, province of Equateur in the DRC}

\begin{abstract}
This study contributes to the inventory of medicinal plants and medicinal recipes used in the treatment of abscesses in the territory of Bikoro, Equateur province in the Democratic Republic of Congo. Named "Matuku" by local populations, abscesses are one of the most common diseases found in Bikoro
\end{abstract}


territory. The lack of adequate sanitary structures or even non-existent health facilities means that Bikoro people resort to traditional medicine for treatment. This motivated the realization of this study. A semistructured interview based upon a questionnaire, in which 32 traditional healers with at least 10 years of experience participated, led to the identification of 43 types of medicinal plants distributed in 39 gender and 27 families. The best represented were Euphorbiacaee (3 gender and 4 types) and Asteraceae (3/3), Commelinaceae (2/2) and Araceae (2/2). Isolated or in mixture, these plants are used in the formulation of 50 medicinal recipes. The leaves and bark of the trunk-stem constitute the principal organs solicited. Preferentially, the preparation of the remedies is done by crushing-pounding and their administration by local application that is to say on the abscess. In relation to their cultural importance, Boerhavia diffusa L., Bridelia micrantha (Hoshst.) Baill. and Psydrax palma (K. Schum.) Bridson are the most recommended.

(C) 2019 International Formulae Group. All rights reserved.

Keywords: Medicinal plants, ethnobotany, abscesses, Democratic Republic of Congo.

\section{INTRODUCTION}

Par définition, un abcès est une accumulation localisée de pus dans une cavité (Safdar et al., 2008). Il peut être superficiel ou profond (Safdar et al., 2008 ; Stevens et al., 2014 ; Talan et al., 2016). Il est provoqué par des agents pathogènes, dits pyogènes (producteurs de pus), introduits le plus souvent par inoculation, comme des bactéries (staphylocoques, streptocoques, pneumocoques...), des parasites, des virus facilitant une surinfection bactérienne, des corps étrangers, des corps chimiques (caustiques), etc. (Safdar et al., 2008 ; Fritz et al., 2009 ; Miller et al., 2009). Sa présence se détecte souvent par les quatre critères rougeur, chaleur, douleur et gonflement (ou œdème) ; l'évacuation du pus est le principal traitement, complété par une antibiothérapie selon les cas (Philippe et al., 2004). Il est assez fréquent dans la population de Bikoro (Ilumbe, 2010) qui le nomme sous l'appellation de «Matuku ». Dans le territoire de Bikoro, ces abcès sont souvent soignés par les tradithérapeutes qui ont développé de multiples thérapies (Ilumbe, 2010).

Dans de nombreux pays tropicaux et surtout en milieu rural comme le territoire de Bikoro, les structures sanitaires sont moins développées voire même inexistantes (Ilumbe, 2010 ; 2014). De plus et malgré l'avènement des médicaments génériques, de nombreux traitements demeurent financièrement encore inaccessibles aux populations économiquement démunies (Ilumbe, 2010 ; Zerbo, 2007). C'est ainsi qu'elles continuent à se tourner vers la nature pour y rechercher des remèdes essentiels à base de plantes. La médecine et la pharmacopée traditionnelles restent alors les seules sources de remède possible.

Pour documenter et ainsi pérenniser ce savoir traditionnel, divers travaux de recherches ethnopharmacologiques ont été entrepris. Certains d'entre eux ont couvert l'Afrique tropicale, l'Afrique sahélienne, l'Afrique de l'Ouest voire l'Afrique entière (Ilumbe, 2010). D'autres travaux ont porté sur des zones plus restreintes dans la sous-région : dans le Dja au Cameroun (Betti, 2001 ; Ilumbe, 2010), en Afrique occidentale ou en Afrique-Centrale (Ilumbe, 2010). En République Démocratique du Congo, un nombre limité de travaux de recherche portant sur l'inventaire et l'utilisation des plantes médicinales ont été menés chez les Bobangi (Ilumbe, 2006) ou à Bikoro (Ilumbe, 2010 ; Ilumbe et al., 2014). Dans la province de l'Equateur, peu de travaux de recherches portant sur l'inventaire et l'utilisation des plantes médicinales ont été menés dans le territoire de Bikoro (Ilumbe, 2010 ; Ilumbe et al., 2014). C'est ainsi que nous avons entrepris une étude dans ce territoire et l'objectif visé était de faire l'inventaire des plantes médicinales et des recettes médicamenteuses utilisées dans le traitement de l'abcès dans le territoire de Bikoro. 


\section{MATERIEL ET METHODES}

\section{Site d'étude et populations étudiées}

Le territoire de Bikoro est situé entre $00^{\circ} 49^{\prime}$ et $1^{\circ} 05^{\prime}$ de latitude Sud et entre $17^{\circ} 55^{\prime}$ et $18^{\circ} 30^{\prime}$ de longitude Est (Figure 1). Il est limité au nord par la ville de Mbandaka (le chef-lieu de la province), au nord-est par le territoire d'Ingende, au sud-est par le territoire de Kiri, au Sud par le territoire d'Inongo, au sud-ouest par le territoire de Lukolela et au nord-ouest par le territoire de Bomongo.

Administrativement, le territoire de Bikoro est subdivisé en 3 secteurs : Elanga, Ekonda et lac Ntomba. Sa population est d'environ 250.000 habitants tandis que sa superficie est de $13.842 \mathrm{~km}^{2}$ avec une densité d'environ 17,68 habitants par $\mathrm{km}^{2}$. D'après les critères de Köppen, le climat de Bikoro est du type Af (climat de la cuvette forestière) où les moyennes mensuelles de précipitation ne sont jamais inférieures à $60 \mathrm{~mm}$ (Trochain, 1980). Ce climat est remarquable par sa pluviosité presque permanente. On compte dix à onze mois très pluvieux (rarement neuf ou douze) avec aucun mois sec (Aubreville, 1949 in Trochain, 1980). Le territoire de Bikoro est caractérisé par un réseau hydrographique très complexe : elle comprend, outre le lac Tumba, appendice du fleuve Congo, les rivières qui s'y déversent, et tout un système de marais drainé par ce même lac. Le lac Tumba est une cuvette de peu de profondeur (3 à $8 \mathrm{~m}$ ), dont les rives sont en majeure partie densément boisées (Marlier, 1958 ; Evrard, 1968). Peu étudié, le sud-ouest de la province de l'Equateur en RDC se rapproche de la forêt de type guinéen défini par Aubreville (Aubreville, 1949), Evrard (Evrard, 1968) et White (White, 1986) : forêt ombrophile équatoriale inondée parsemée de taches de forêts clairsemées.

Le territoire de Bikoro est occupé par des Mongo (plus précisément des Ntomba, des Ekonda et des Elanga) qui parlent des langues bantoues (C60 selon la classification de Guthrie, 1971) et des pygmées Twa (locuteurs des mêmes langues). Les ba-Oto (Mongo non pygmées) sont des agriculteurs-pêcheurs tandis que les Mongo pygmées Twa sont des chasseurs-cueilleurs. Toutefois, les deux groupes pratiquent également la chasse et la cueillette pour les premiers, l'agriculture et la pêche pour les seconds. A Bikoro, les Twa et leur voisin Oto vivent fixés dans les mêmes villages depuis de nombreuses générations, exploitent les mêmes milieux, malgré qu'ils se différencient par leur identité et les modalités d'exploitation des ressources naturelles.

\section{Méthodes}

La présente étude a été réalisée à partir d'une série d'enquêtes ethnobotaniques utilisant l'entretien semi- directif à partir d'un questionnaire pré-élaboré et les renseignements recherchés ont porté sur les plantes qu'ils utilisent pour traiter l'abcès, les organes de la plante utilisés, le mode de préparation des recettes et le mode d'administration (Martin, 1995 ; Kalanda et al., 1995 ; Ilumbe, 2006 ; Alexiades, 1996 ; Zerbo et al., 2007). Les questions ont été posées dans la langue locale, le lingala. Au total, 32tradipraticiens de santé et herboristes ont accepté de collaborer avec nous. Ils avaient tous une expérience d'au moins 15 ans dans l'utilisation des plantes en médecine et pharmacopée traditionnelles. Lors des enquêtes, nous avons insisté sur les recettes concernant l'abcès. Cinq (05) des enquêtés dont 3 infirmiers ont participé à l'identification formelle de la maladie et des plantes.

Comme il s'agit des guérisseurs, il nous a fallu se conformer aux pratiques locales pour les aborder. Avant l'entretien, il faut avoir une bouteille de boisson alcoolisée « lotoko » accompagnée souvent d'un paquet de cigarettes. Après l'entretien et avant de passer à la récolte des échantillons, il faut donner une petite somme équivalente de 1 dollar selon le cas. Ce geste appelé communément «Ikondela Ngonda », qui signifie « autorisation ou le droit d'entrer dans la forêt. 
Une technique qui prend en compte le consensus de participants et peut ainsi être utilisée pour évaluer l'importance culturelle des plantes est la proportion d'accord d'informateur (Trotter et Logan, 1986 ; Thomas et al., 2009). Pour la présente étude, nous avons interprété la formule d'origine proposée par Byg et Balslev (Byg et Balslev, 2001), pour exprimer le consensus d'informateurs ou l'indice de confirmation des plantes médicinales. Ainsi, le consensus d'informateurs se calcule comme suit :

$$
I C s=\frac{N a}{N t}(1)
$$

Où ICs est l'indice de confirmation, $\mathrm{Na}=$ nombre de personnes ayant cité cette espèce et $N t=$ nombre total de personnes interviewées.

L'identification scientifique des espèces a été réalisée sur le terrain. Les échantillons que nous n'avons pas pu déterminer sur place ont été ramenés à l'herbarium du jardin botanique d'Eala et du Département de Biologie de l'Université de Kinshasa pour l'identification par comparaison. Ces herbaria possèdent une bonne collection des plantes tropicales. La nomenclature botanique suivie est celle reprise dans les quatre volumes de Lebrun \& Stork (Lebrun et al., 1991-1997), le deuxième volume de la nouvelle édition de Lebrun \& Stork (Lebrun et al., 2006), et la base de données en ligne sur www.villege.ch/cjb/bd/Africa/index.php/francce. Les familles ont été classées sur base du système APG III 2009 (Angiosperm Phylogeny Group) (Elspeth et al., 2009).

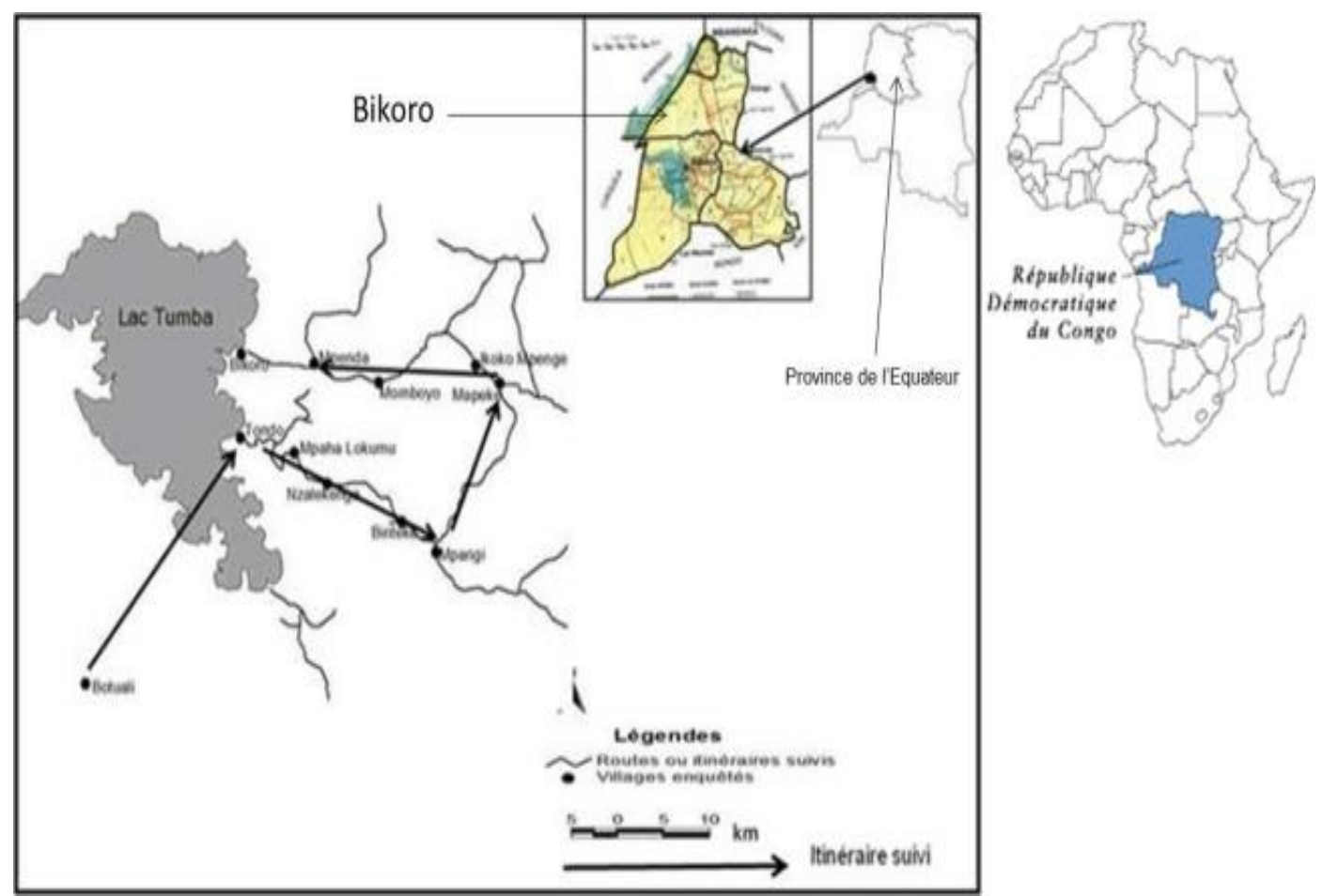

Figure 1 : Carte administrative du territoire de Bikoro et l'itinéraire suivi. (Source : Ilumbe Bayeli G). 


\section{RESULTATS}

Au total, nous avons identifié 43 plantes médicinales (Tableau 1) réparties en 39 genres et 27 familles. Ces espèces interviennent dans la confection de plus ou moins 50 recettes médicamenteuses.

Les plantes recensées sont composées de 20 herbes vivaces soit $46,5 \%$. Elles sont suivies par les arbustes ( 8 espèces soit 18,6\%), les arbres (7espèces soit 16,3\%), les lianes (6 espèces soit $14 \%$ ) et 2 sous-arbustes soit $4,7 \%$ (Figure 2).

Les feuilles, les écorces (tige et tronc), les fruits ainsi que les organes souterrains (bulbe, tubercule, et racine) sont les parties utilisées. Les feuilles prédominent avec une fréquence de citation de $46,8 \%$ de citations. Elles sont suivies par les écorces (tige et tronc) avec 31,2\% de citations (Figure $3)$.

Le pilage et trituration $(32 \%$ de recettes), le ramollissement au feu (18\% de recettes), l'extraction ( $16 \%$ de recettes) sont les modes de préparation des recettesles plus employés. $11 \%$ de recettes sont sans préparation; il s'agit juste de l'extraction de la sève à appliquer directement sur les abcès. En ce qui concerne le mode d'administration de recettes, $92 \%$ de recettes appartenant à 40 espèces sont appliquées localement sur les abcès.Seules les recettes provenant de Pyrenacantha staudtii (Engl.) Engl., Oncoba welwitschii De Wild. \& Th. Dur. et Pyrenacantha staudtii (Engl.) Engl. (soit 6\%) sont employées par instillation oculaire et $2 \%$ de recettes par voie orale.

$\mathrm{Au}$ total, 178 citations ont été recueillies. Les espèces Boerhavia diffusa L., Bridelia stenocarpa Müll. Arg., Psydrax palma (K. Schum.) Bridson, Dracaena arborea (Willd.) Link, Gilbertiodendron dewevrei (De Wild.) J. Léonard, Acanthus montanus T. Anders., Cnestis ferruginea DC. et Tetrorchidium sp. sont les plus citées par les informateurs (Tableau 2).

Tableau 1 : Nombre de genres et d'espèces par famille.

\begin{tabular}{lcc}
\hline Familles & $\begin{array}{c}\text { Nombre } \\
\text { de genre }\end{array}$ & $\begin{array}{c}\text { Nombre } \\
\text { d'espèces }\end{array}$ \\
\hline Euphorbiaceae & 3 & 4 \\
\hline Asteraceae & 3 & 3 \\
\hline Commelinaceae & 2 & 3 \\
\hline Araceae & 2 & 2 \\
\hline Asparagaceae & 1 & 2 \\
\hline Flacourtiaceae & 2 & 2 \\
\hline Icacinaceae & 1 & 2 \\
\hline Lamiaceae & 2 & 2 \\
\hline Malvaceae & 2 & 2 \\
\hline Piperaceae & 1 & 2 \\
\hline Rubiaceae & 2 & 2 \\
\hline Urticaceae & 2 & 2 \\
\hline Phyllanthaceae & 2 & 2 \\
\hline Acanthaceae, Cannaceae, Caricaceae, Connaraceae, & & \\
Convolvulaceae, Cucurbitaceae, Fabaceae, Maranthaceae, & & \\
Moraceae, Musaceae, Nyctaginaceae, Passifloraceae, Solanaceae, & & 1 \\
Sterculiaceae, Urticaceae, Vitaceae & 1 & $\mathbf{4 3}$ \\
\hline Total = 27 & $\mathbf{3 9}$ & \\
\hline
\end{tabular}




\section{Les différentes formes biologiques des plantes utilisées}

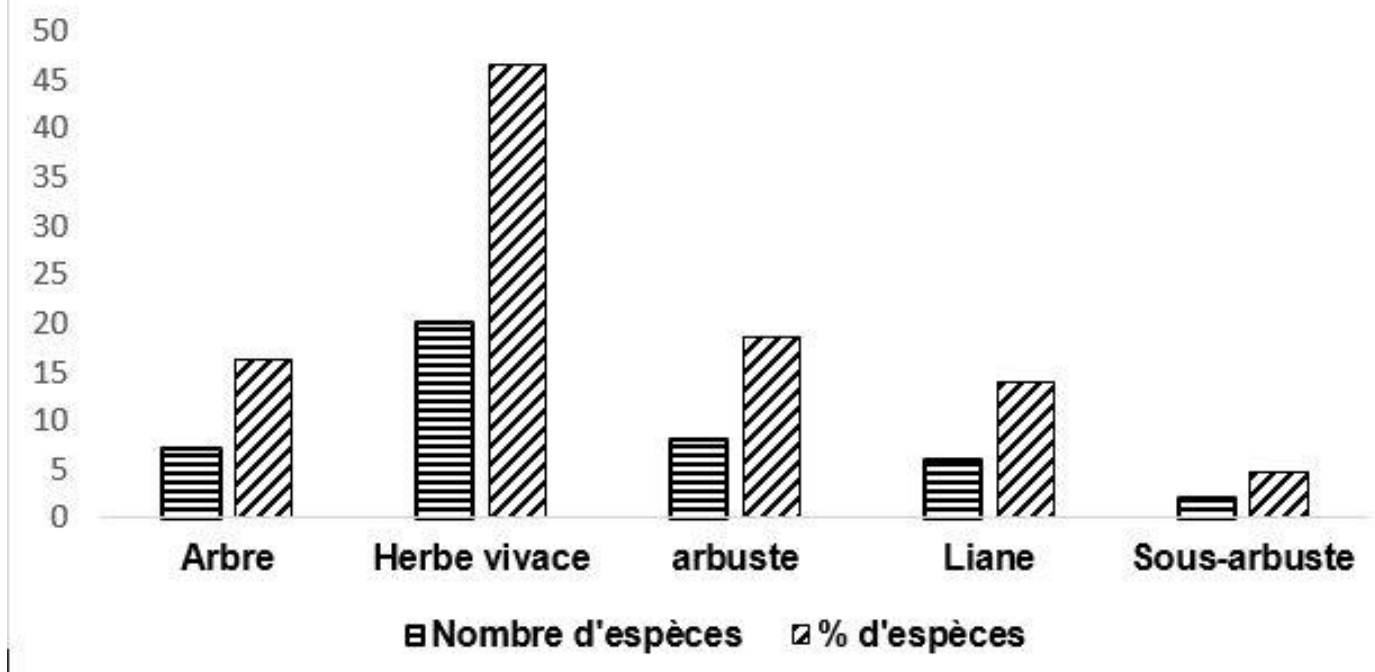

Figure 2 : Nombre et fréquence de types morphologiques de plantes utilisées contre les abcès dans le territoire de Bikoro.

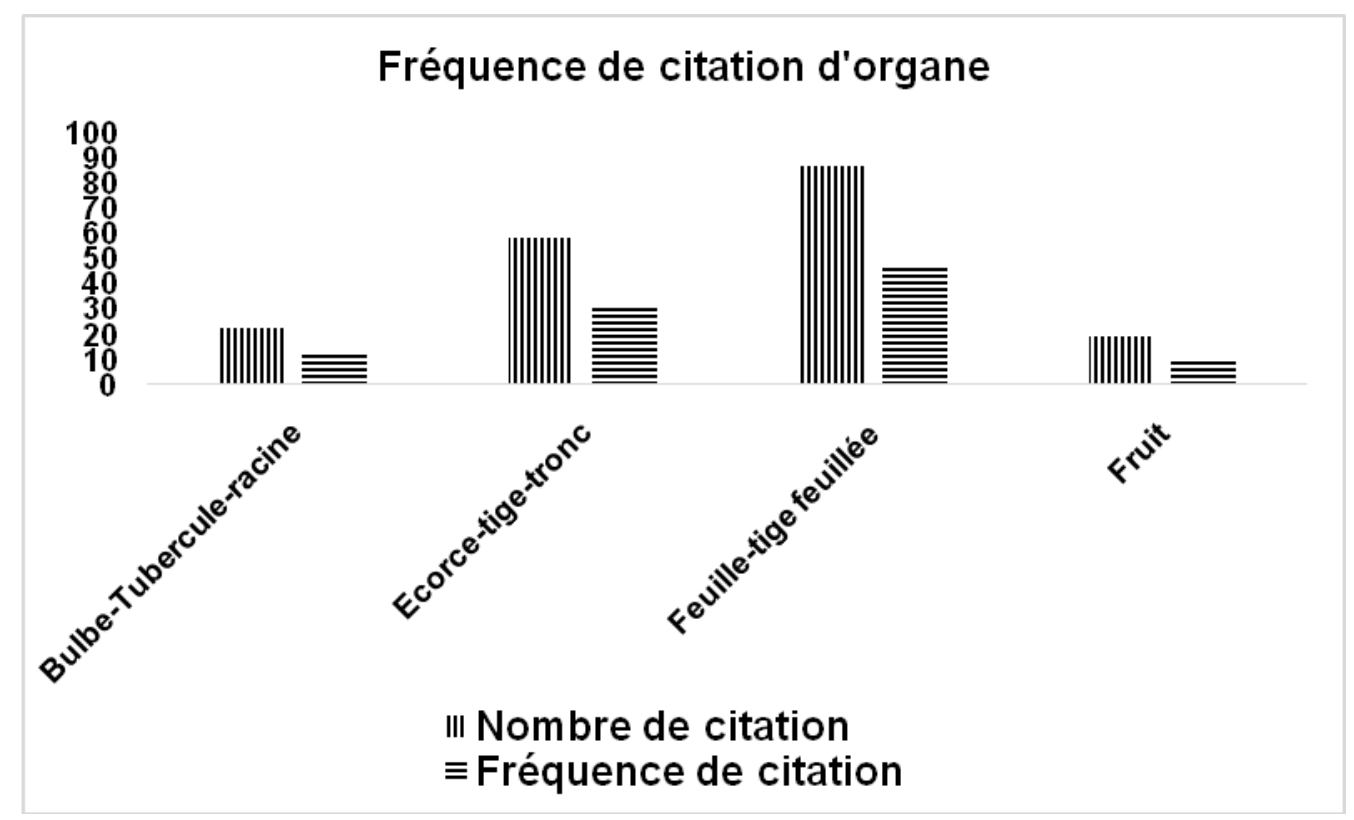

Figure 3: Nombre et fréquence de citation des organes de plantes utilisés. 
Tableau 2 : Liste des plantes utilisées dans le traitement des abcès ayant une valeur de l'indice de confirmation supérieure à 0,10 .

\begin{tabular}{|c|c|c|c|}
\hline Espèces & N.C & $\% . \mathrm{C}$ & Ic \\
\hline Boerhavia diffusa $\mathrm{L}$. & 12 & 6,45 & $\mathbf{0 , 3 8}$ \\
\hline Brideliamicrantha (Hoshst.) Baill. & 12 & 6,45 & $\mathbf{0 , 3 8}$ \\
\hline Psydrax palma (K. Schum.) Bridson & 10 & 5,38 & $\mathbf{0 , 3 1}$ \\
\hline Dracaena arborea (Willd.) Link & 9 & 4,84 & 0,28 \\
\hline Gilbertiodendrondewevrei (De Wild.) J.Léonard & 9 & 4,84 & 0,28 \\
\hline Acanthusmontanus T. Anders. & 8 & 4,30 & 0,25 \\
\hline Cnestisferruginea DC. & 8 & 4,30 & 0,25 \\
\hline Tetrorchidiumsp. & 8 & 4,30 & 0,25 \\
\hline Ageratum conyzoides $\mathrm{L}$. & 7 & 3,76 & 0,22 \\
\hline HymenocardiaulmoidesOliv. & 7 & 3,76 & 0,22 \\
\hline MicrodesmispuberulaHook.f. ex Planch. & 7 & 3,76 & 0,22 \\
\hline Laporteaaestuans (L.) Chew. & 6 & 3,23 & 0,19 \\
\hline CogniauxiatrilobataCogn. & 6 & 3,23 & 0,19 \\
\hline Pyrenacanthastaudtii(Engl.) Hutch. Et Dalz. & 5 & 2,69 & 0,16 \\
\hline CaloncobawelwitschiiDe Wild. \& Th. Dur. & 5 & 2,69 & 0,16 \\
\hline AdenostemmaviscosumForst. & 5 & 2,69 & 0,16 \\
\hline Caladium bicolor(Aiton) Vent. & 5 & 2,69 & 0,16 \\
\hline CaricapapayaL. & 4 & 2,15 & 0,13 \\
\hline Lindackeriadentata(Oliv.) Gilb. & 4 & 2,15 & 0,13 \\
\hline
\end{tabular}

Légende : N.C = nombre de citation ; \%C = pourcentage de citation, Ic = indice de confirmation.

Tableau 3 : Liste des familles et des plantes utilisées dans le traitement des abcès dans le territoire de Bikoro.

\begin{tabular}{|c|c|c|c|c|c|}
\hline Familles/espèces & $\begin{array}{c}\text { Type } \\
\text { Morphologique }\end{array}$ & $\begin{array}{l}\text { Partie } \\
\text { utilisée }\end{array}$ & $\begin{array}{c}\text { Mode de } \\
\text { préparation }\end{array}$ & $\begin{array}{c}\text { Mode } \\
\text { d'administration } \\
\end{array}$ & $\begin{array}{l}\text { Travaux } \\
\text { cités }\end{array}$ \\
\hline \multicolumn{6}{|l|}{ Acanthaceae } \\
\hline Acanthusmontanus T. Anders. & Sarb & Tige & Trituration & $\begin{array}{l}\text { Application } \\
\text { locale }\end{array}$ & $\mathrm{T} 1, \mathrm{~T} 3$ \\
\hline \multicolumn{6}{|l|}{ Araceae } \\
\hline AnchomanesgiganteusEngl. & $\mathrm{Hv}$ & Tubercule & Pilage & $\begin{array}{c}\text { Application } \\
\text { locale }\end{array}$ & $\mathrm{T} 1, \mathrm{~T} 2$ \\
\hline \multirow[t]{3}{*}{ Caladium bicolor (Aiton) Vent. } & $\mathrm{Hv}$ & Bulbe & Pilage & $\begin{array}{l}\text { Application } \\
\text { locale }\end{array}$ & $\mathrm{T} 1, \mathrm{~T} 3$ \\
\hline & & & Trituration & $\begin{array}{c}\text { Application } \\
\text { locale }\end{array}$ & $\mathrm{T} 1, \mathrm{~T} 3$ \\
\hline & & Tubercule & Trituration & $\begin{array}{l}\text { Application } \\
\text { locale }\end{array}$ & $\mathrm{T} 1, \mathrm{~T} 3$ \\
\hline \multicolumn{6}{|l|}{ Asparagaceae } \\
\hline Dracaena arborea (Willd.) Link & A & Ecorce tige & Trituration & Application & $\mathrm{T} 1, \mathrm{~T} 3$ \\
\hline
\end{tabular}




\begin{tabular}{lccccc}
\hline & & & & locale \\
\hline Dracaena mannii Baker & A & Ecorce tige & Trituration & $\begin{array}{c}\text { Application } \\
\text { locale }\end{array}$ & $\mathrm{T} 1, \mathrm{~T} 3$ \\
\hline Asteraceae & & & & & \\
\hline Adenostemmaviscosum Forst. & $\mathrm{Hv}$ & Feuille & Pilage & $\begin{array}{c}\text { Application } \\
\text { locale }\end{array}$ & $\mathrm{T} 1, \mathrm{~T} 2$ \\
\hline Ageratum conyzoides L. & $\mathrm{Hv}$ & Feuille & $\begin{array}{c}\text { Ramollissement } \\
\text { au feu }\end{array}$ & $\begin{array}{c}\text { Application } \\
\text { locale }\end{array}$ & $\mathrm{T} 1, \mathrm{~T} 2$ \\
\hline Mikaniascandens $\mathrm{W}$. & Sarb & $\begin{array}{c}\text { Sève de la } \\
\text { feuille }\end{array}$ & Pilage & $\begin{array}{c}\text { Application } \\
\text { locale }\end{array}$ & $\mathrm{T} 1, \mathrm{~T} 2$ \\
\hline
\end{tabular}

\section{Cannaceae}

\begin{tabular}{lccccc}
\hline & & & Ramollissement & Application \\
Canna indica $\mathrm{L}$. & $\mathrm{Hv}$ & Feuille & au feu & locale & $\mathrm{T} 1, \mathrm{~T} 3$ \\
\hline
\end{tabular}

\section{Caricaceae}

\begin{tabular}{llllll}
\hline & & & & & Application \\
Caricapapaya L. & A & Fruit & Trituration & locale & T1, T3 \\
\hline
\end{tabular}

\section{Commelinaceae}

\begin{tabular}{|c|c|c|c|c|c|}
\hline & & & & Application & \\
\hline Aneilemasp. & $\mathrm{Hv}$ & Tige & Pilage & locale & $\mathrm{T} 1$ \\
\hline PalisotabrachythyrsaMildbr. & $\mathrm{Hv}$ & Feuille & $\begin{array}{c}\text { Ramollissement } \\
\text { au feu }\end{array}$ & $\begin{array}{l}\text { Application } \\
\text { locale }\end{array}$ & $\mathrm{T} 1, \mathrm{~T} 3$ \\
\hline Palisotahirsuta (Thunb.) K.Schum. & $\mathrm{Hv}$ & Feuille & $\begin{array}{c}\text { Ramollissement } \\
\text { au feu }\end{array}$ & $\begin{array}{l}\text { Application } \\
\text { locale }\end{array}$ & $\mathrm{T} 1$ \\
\hline
\end{tabular}

\section{Connaraceae}

Cnestisferruginea DC.

$\mathrm{L}$

Feuille

\section{Application} locale

\section{Convolvulaceae}

Ipomoeadigitata $\mathrm{L}$.

Hv

$\begin{array}{ll}\text { Jeune } & \\ \text { pousse } & \text { Pilage }\end{array}$

Application
locale

\section{Cucurbitaceae}

\begin{tabular}{|c|c|c|c|c|c|}
\hline CogniauxiatrilobataCogn. & $\mathrm{Hv}$ & Tubercule & Pilage & $\begin{array}{l}\text { Application } \\
\text { locale }\end{array}$ & $\mathrm{T} 1, \mathrm{~T} 2$ \\
\hline \multicolumn{6}{|l|}{ Euphorbiaceae } \\
\hline $\begin{array}{l}\text { MicrodesmispuberulaHook.f. ex } \\
\text { Planch. }\end{array}$ & arb & $\begin{array}{l}\text { Sève de la } \\
\text { feuille }\end{array}$ & Extraction & $\begin{array}{l}\text { Application } \\
\text { locale }\end{array}$ & $\mathrm{T} 1$ \\
\hline $\begin{array}{l}\text { Tetrorchidiumdidymostemon } \\
\text { (Baill.) Pax et Hoffm. }\end{array}$ & arb & $\begin{array}{l}\text { Sève de la } \\
\text { tige }\end{array}$ & Extraction & $\begin{array}{l}\text { Application } \\
\text { locale }\end{array}$ & $\mathrm{T} 1$ \\
\hline Tetrorchidiumsp & arb & Ecorce tige & Trituration & $\begin{array}{l}\text { Application } \\
\text { locale }\end{array}$ & $\mathrm{T} 1$ \\
\hline Tragiasp. & $\mathrm{Hv}$ & Fruit & $\begin{array}{c}\text { Sans } \\
\text { préparation }\end{array}$ & $\begin{array}{l}\text { Application } \\
\text { locale }\end{array}$ & $\mathrm{T} 1$ \\
\hline
\end{tabular}


\begin{tabular}{l} 
Fabaceae \\
\hline Gilbertiodendrondewevrei (De \\
Wild.) J.Léonard
\end{tabular}

\section{Flacourtiaceae}

Oncobawelwitschii De Wild. \& Th.

Dur.

Lindackeriadentata (Oliv.) Gilb.

\section{Icacinaceae}

Pyrenacanthastaudtii (Engl.) Engl.

L

arb

Sève tige

Extraction

Application

locale

$\mathrm{T} 1, \mathrm{~T} 3$

Feuille au feu locale $\quad$ T3

Feuille Trituration Instilloc

Jeune

$\mathrm{T} 1, \mathrm{~T} 3$

pousse $\quad$ Extraction $\quad$ Instilloc $\quad$ T1, T3

\begin{tabular}{llllll}
\hline & L & Feuille & Trituration & Instilloc & T1 \\
\hline
\end{tabular}

\section{Lamiaceae}

\begin{tabular}{|c|c|c|c|c|c|}
\hline HyptisspicigeraLam. & $\mathrm{Hv}$ & Feuille & Pilage & $\begin{array}{c}\text { Application } \\
\text { locale }\end{array}$ & $\mathrm{T} 1, \mathrm{~T} 2$ \\
\hline & & & Trituration & \multicolumn{2}{|c|}{ Application locale } \\
\hline Ocimumcanum $\mathrm{L}$. & $\mathrm{Hv}$ & Feuille & Pilage & \multicolumn{2}{|c|}{ Application locale } \\
\hline \multicolumn{6}{|l|}{ Malvaceae } \\
\hline $\begin{array}{l}\text { Clappertoniapolyandra (K. Schum. } \\
\text { ex Sprague) Bech. }\end{array}$ & $\mathrm{Hv}$ & Racine & $\begin{array}{c}\text { Sans } \\
\text { préparation }\end{array}$ & $\begin{array}{l}\text { Application } \\
\text { locale }\end{array}$ & $\mathrm{T} 1$ \\
\hline Sida rhombifolia $\mathrm{L}$. & $\mathrm{Hv}$ & Feuille & Pilage & $\begin{array}{l}\text { Application } \\
\text { locale }\end{array}$ & $\mathrm{T} 1$ \\
\hline \multicolumn{6}{|l|}{ Maranthaceae } \\
\hline HaumaniaLiebrechtsiana K. Schum & $\mathrm{Hv}$ & Graines & Pilage & $\begin{array}{l}\text { Application } \\
\text { locale }\end{array}$ & $\mathrm{T} 1, \mathrm{~T} 3$ \\
\hline \multicolumn{6}{|l|}{ Musaceae } \\
\hline Musa sinensisSag. & arb & Fruit & Pilage & $\begin{array}{l}\text { Application } \\
\text { locale }\end{array}$ & $\mathrm{T} 1, \mathrm{~T} 3$ \\
\hline \multicolumn{6}{|l|}{ Nyctaginaceae } \\
\hline Boerhavia diffusa $\mathrm{L}$. & $\mathrm{Hv}$ & Feuille & $\begin{array}{c}\text { Ramollissement } \\
\text { au feu }\end{array}$ & $\begin{array}{l}\text { Application } \\
\text { locale }\end{array}$ & $\mathrm{T} 1, \mathrm{~T} 2, \mathrm{~T} 3$ \\
\hline & & & $\begin{array}{c}\text { Sans } \\
\text { préparation }\end{array}$ & Applicatic & locale \\
\hline
\end{tabular}

\section{Passifloraceae}

Adenialobata (Jacq.) Engl.

$\mathrm{L}$

Ramollissement Application 


\begin{tabular}{|c|c|c|c|c|c|}
\hline Phyllanthacace & & & & & \\
\hline & & & & Application & \\
\hline HymenocardiaulmoidesOliv. & A & Racine & Pilage & locale & $\mathrm{T} 1$ \\
\hline & & & & Application & \\
\hline Brideliamicrantha(Hochst.) Baill. & arb & Sève tige & Extraction & locale & $\mathrm{T} 1$ \\
\hline Piperaceae & & & & & \\
\hline Piper guineensisSchum. \&Thonn. & $\mathrm{L}$ & Feuille & $\begin{array}{c}\text { Ramollissement } \\
\text { au feu }\end{array}$ & $\begin{array}{l}\text { Application } \\
\text { locale }\end{array}$ & $\mathrm{T} 1$ \\
\hline Piper umbellatum $\mathrm{L}$. & $\mathrm{Hv}$ & Feuille & $\begin{array}{c}\text { Sans } \\
\text { préparation }\end{array}$ & $\begin{array}{l}\text { Application } \\
\text { locale }\end{array}$ & $\mathrm{T} 1, \mathrm{~T} 2, \mathrm{~T} 3$ \\
\hline Rubiaceae & & & & & \\
\hline Psydrax palma (K. Schum.) & & & & & \\
\hline Bridson & arb & Fruit & Extraction & Per os & $\mathrm{T} 1$ \\
\hline Morindamorindoides (Baker) & & & & & \\
\hline Milne-Redh. & $\mathrm{L}$ & Feuille & Extraction & Instilloc & $\mathrm{T} 1$ \\
\hline Solanaceae & & & & & \\
\hline Capsicumfrutescens $\mathrm{L}$. & $\mathrm{Hv}$ & Feuille & $\begin{array}{c}\text { Ramollissement } \\
\text { au feu }\end{array}$ & $\begin{array}{l}\text { Application } \\
\text { locale }\end{array}$ & $\mathrm{T} 1, \mathrm{~T} 2, \mathrm{~T} 3$ \\
\hline Sterculiaceae & & & & & \\
\hline Cola acuminata (P.B.) Schott et & & & & Application & \\
\hline Endl. & A & Ecorce tige & Pilage & locale & $\mathrm{T} 1$ \\
\hline
\end{tabular}

\begin{tabular}{|c|c|c|c|c|c|}
\hline \multicolumn{6}{|l|}{ Urticaceae } \\
\hline \multirow[b]{2}{*}{ Laporteaaestuans (L.) Chew. } & \multirow[b]{2}{*}{$\mathrm{Hv}$} & \multirow[b]{2}{*}{ Feuille } & \multicolumn{3}{|c|}{ Application } \\
\hline & & & Trituration & locale & $\mathrm{T} 1, \mathrm{~T} 2, \mathrm{~T} 3$ \\
\hline & & $\begin{array}{l}\text { Sève de la } \\
\text { feuille }\end{array}$ & Extraction & \multicolumn{2}{|c|}{ Application locale } \\
\hline & & Jeune & & Application & \\
\hline Myrianthusarboreus P. Beauv & A & pousse & Pilage & locale & $\mathrm{T} 1, \mathrm{~T} 3$ \\
\hline \multicolumn{6}{|l|}{ Vitaceae } \\
\hline Cissusaralioides & $\mathrm{L}$ & Feuille & Trituration & $\begin{array}{l}\text { Application } \\
\text { locale }\end{array}$ & $\mathrm{T} 1$ \\
\hline
\end{tabular}




\section{DISCUSSION}

La famille Euphorbiaceae est la plus représentée avec 4 genres et 4 espèces. Elle est suivie par la famille Asteraceae avec 3 genres et 3 espèces et celle des Commelinaceae avec 2 genres et 3 espèces. Le caractère pérenne de ces espèces permettant de disposer d'au moins un organe en chaque saison pourrait justifier cette sollicitude. Olivier et al. (2003), Nana-Sanon (2005) et Ilumbe et al. (2014) ont également constaté cette forte utilisation des espèces ligneuses en pharmacopée traditionnelle.

Le classement fait à partir des valeurs de l'indice de confirmation (ICs) met en première position les espèces Boerhavia diffusa L., Bridelia stenocarpa Müll.Arg., Psydrax palma (K. Schum.) Bridson, Dracaena arborea (Willd.) Link, Gilbertiodendron dewevrei (De Wild.) J.Léonard, Acanthus montanus T. Anders., Cnestis ferruginea DC. et Tetrorchidium $s p$. avec des valeurs supérieures ou égales à 0,25 . (Tableau 2).

Sur l'ensemble de 43 espèces inventoriées pour les soins des abcès dans le territoire de Bikoro (voir tableau 3), 44,04\% constituent la contribution de la présente étude. Par contre, $11,5 \%$ d'espèces ont été citées par Ilumbe (2010), 32,6\% par (Hulstaert, 1966 in Ilumbe, 2010) et $11,5 \%$ par Ilumbe (2010) et (Hulstaert, 1966 in Ilumbe, 2010).

En Inde, Boerhavia diffusa est une plante médicinale très prisée; ce sont surtout ses racines, ses feuilles et ses graines qui sont utilisées et les racines figurent dans la pharmacopée indienne (Satheesh et al., 2004 ; Slamet Sutanti et al., 2001). Une décoction de feuilles et le jus frais ont tous deux eu un effet analgésique important lors d'essais chez les rats, mais le jus frais a relevé le seuil nociceptif beaucoup plus longtemps que la décoction de feuilles. L'extrait alcoolique a montré des effets anti-inflammatoires contre l'œdème de la patte provoqué par le carraghénane et a augmenté la diurèse chez le rat (Satheesh et al., 2004). Une décoction de feuilles sert en R.D. du Congo à traiter la gonorrhée et à soulager la douleur (Kibungu, 2004 ; Latham, 2004). Carie dentaire, Panaris (Ilumbe, 2010).

$\mathrm{Au}$ Congo-Brazzaville, la décoction d'écorce de Bridelia micrantha (Hoshst.) Baill. se prend en cas de toux et de maux de gorge (Bitsindou, 1997 in Ilumbe, 2010). En R.D. du Congo, l'écorce interne sert à confectionner un poison de flèche. Contre les maux de tête (Ilumbe, 2010). Les tanins isolés de l'écorce de Bridelia micrantha ont montré une activité antibactérienne, et les extraits aqueux de l'écorce ont mis en lumière un effet anti-inflammatoire (Bessong et al., 2005). Il a été établi que des extraits méthanoliques et aqueux des racines et de l'écorce de la tige de Bridelia micrantha avaient une puissante activité contre l'intégrase et la transcriptase inverse du VIH-1 (Bessong et al., 2005). Des extraits méthanoliques de l'écorce ont montré in vitro l'inhibition d'un grand nombre de bactéries gram-positives et gram-négatives. Des extraits de racine ont eu le même effet mais seulement à des concentrations plus élevées. Parmi les composés isolés qui sont probablement responsables de l'activité antibactérienne, figurent la friedéline, la taraxérone, l'épifriedélinol, le taraxérol ainsi que des tanins (l'acide gallique, l'acide ellagique et l'acide caféique (Gangoué-Piéboji et al., 2005 ; 2006). En effet, plusieurs auteurs ont montré que les types de composés chimiques mis en évidence dans les extraits de cette plante ont des effets thérapeutiques (Nene Bi et al., 2009). Dans la région d'étude, Dracaena arborea est citée contre la Hernie, les Maux de yeux, la Panaris et la Rougeole (Ilumbe, 2010 ; 2006), Gilbertiodendron 
dewevrei est utilisée contre la diarrhée, la Gastrite et la blennorragie (Ilumbe, 2010 ; Terashima, 2003; http://www.prota.org), Tetrochydium $s p$ est employé aussi contre Carie dentaire, Cicatrisation des plaies, Constipation (Ilumbe, 2010) et comme purgatif (Hulstaert, 1966 in Ilumbe, 2010). Oncoba welwitschii est utilisée aussi contre les Cataractes, la Conjonctivite, la Diarrhée, l'Epilepsie, les maux d'estomac, pour faciliter la grossesse, contre les hémorroïdes, Maux de tête, Pneumonie (Hulstaert, 1966 in Ilumbe, 2010 ; Ilumbe, 2006, 2010).

\section{Conclusion}

L'enquête réalisée dans le territoire de Bikoro, province de l'Equateur en République Démocratique du Congo a permis de recenser 43 espèces utilisées dans le traitement des abcès. Les espèces identifiées sont réparties en 39 genres et 27 familles. Leurs actions médicales ont été vérifiées par plusieurs expériences traditionnelles à en croire nos informateurs. Des essais biologiques et chimiques permettront de vérifier la véracité des indications thérapeutiques reçues.

La diversité des propriétés et des indications, les divers organes utilisés ainsi que les modes de préparation et d'administration, témoignent d'une bonne connaissance de la diversité végétale des tradipraticiens du territoire de Bikoro. Les feuilles, les écorces constituent les organes les mieux utilisés pour les soins des abcès. Après leur extraction, le problème de la survie des espèces se pose. Pour une meilleure utilisation des plantes médicinales, tout en préservant les ressources naturelles, une politique de sensibilisation des utilisateurs doit être élaborée en vue de leur enseigner les bonnes pratiques de récolte des plantes, la gestion et la protection des plantes in situ et les notions sur les techniques de culture des plantes médicinales. Pour réduire les risques de disparition, il est impératif d'entreprendre l'écologie des espèces très sollicitées. Ainsi les espèces rares ou en voie de disparition, trouveront une solution de survie par la régénération. La complexité du domaine de la médecine traditionnelle a fait perdre des recettes suite à la disparition du détenteur du secret. Des études sur la connaissance des plantes médicinales d'autres territoire sont à encourager afin d'archiver ces informations.

\section{CONFLIT D'INTERETS}

Les auteurs déclarent qu'ils n'ont aucun conflit d'intérêts.

\section{CONTRIBUTIONS DES AUTEURS}

GIB a conçu la méthodologie, analysé les données et contribué à la rédaction du manuscrit. GNL et J-PH ont contribué à l'identification des spécimens. VJ a contribué à l'analyse de données et la rédaction du manuscrit.

\section{REMERCIEMENTS}

La réalisation de cette étude a été possible grâce aux conseils et à la collaboration des tradipraticiens de santé et herboristes suivants: $\mathrm{Mr}$ Charles Mogulu (Ethnobotaniste à la retraite, Jardin Botanique d'EALA, Mbandaka), Mr Lobwano et $\mathrm{Mr}$ Yatshi (Tradipraticiens-Herboristes), Mrs Joseph Mongu, Louis Mpia et Paul Ilanga (Infirmiers retraités). A tous, nous leur adressons nos sincères remerciements de même qu'à tous les tradipraticiens de santé et herboristes qui ont accepté partager leurs connaissances.

\section{REFERENCES}

Alexiades MN. 1996. Selected Guidelines for Ethno Botanical Research: A Field Manual. The New York Botanical Garden: New York. 
Alexiades MN. 1999. Ethnobotany of the EseEja: Plants, health, and change in an Amazonian society. Ph.D. Dissertation, City University of New York.

Aubreville A. 1949. Climats, forêts et désertification. Société d'Editions géologiques, maritimes et coloniales: Paris; 351p.

Bastien JW. 1987. Healers of the Andes. Kallawaya Herbalists and their Medicinal Plants. University of Utah Press: Salt Lake City, US.

Bessong, PO, Obi CL, Andréola ML, Rojas LB, Pouységu L, Igumbor E, Marion Meyer JJ, Quideau S, Litvak S. 2005. Evaluation of selected South African medicinal plants for inhibitory properties against human immunodeficiency virus type 1 reverse transcriptase and integrase. Journal of Ethnopharmacology, 99: 83-91. DOI: https://doi.org/10.1016/j.jep.2005.01.056

Betti JL. 1996. Les plantes médicinales utilisées par les guérisseurs spécialisés dans la réserve de faune du Dja (Cameroun). Projet Ecofac, Agreco-Ctft, Bruxelles, $126 \mathrm{p}$.

Betti JL. 2001. Vulnérabilité des plantes utilisées comme antipaludiques dans l'arrondissement de Mintom au sud de la réserve de biosphère du Dja (Cameroun). Systematics and Geography of Plants, 71(2): $\quad$ 661-678. DOI: 10.2307/3668709

Byg A, Balslev H. 2001. Diversity and use of palms in Zahamena, eastern Madagascar. Biodiversity and Conservation, 10: 951970.

DOI: https://doi.org/10.1023/A:101664071364 3

Elspeth H, James R, Peter F, Mark WC, David JH. 2009. The Linear Angiosperm Phylogeny Group (LAPG) III: a linear sequence of the families in APG III. Botanical Journal of the Linnean, Society, 161: 128-131. DOI: https://doi.org/10.1111/j.10958339.2009.01000.x

Evrard C. 1968. Recherche écologique sur le peuplement forestier de sol hydromorphe de la Cuvette Centrale Congolaise. Sér. Scientif. Publ. : INEAC; 295 p.

Fritz SA, Bininda-Emonds ORP, Purvis A. 2009. Geographical variation in predictors of mammalian extinction risk: Big is bad, but only in the tropics. Ecology Letters, 12: 538-549.

Gangoué-Piéboji J, Baurin S, Frère JM, Ngassam P, Ngameni B, Azebaze A, Pegnyemb DE, Watchueng J, Goffin C, Galleni M. 2007. Screening of some medicinal plants from Cameroon for BLactamase inhibitory activity. Phytotherapy Research, 21(3): 284-287. DOI: https://doi.org/10.1002/ptr.2001

Gangoué-Piéboji J, Pegnyemb DE, Niyitegeka D, Nsangou A, Eze $\mathrm{N}$, Minyem C, Mbing JN, Ngassam P, Tih RG, Sodengam BL, Bodo B. 2006. The in-vitro antimicrobial activities of some medicinal plants from Cameroon. Annals of Tropical Medicine and Parasitology, 100(3): 237-243. DOI: $10.1179 / 136485906 \times 86365$

Guthrie M. 1967. The classification of the Bantu languages. International African Institute, London. 91p.

Guthrie M. 1971. Comparative Bantu: an introduction to the comparative linguistics and prehistory of the Bantu languages. London: Gregg International.

Heinrich M, Ankli A, Frei B, Weimann C, Sticher O. 1998. Medicinal plants in Mexico: Healers' consensus and cultural importance. Social Science and Medicine, 47: 1859-1871. 
Hulstaert G. 1961. Les Mongo ; aperçu générale. Musée Royale de l'Afrique centrale, Tervuren, Belgique, Archives d'ethnographie, 5: 66.

Hulstaert G. 1966. Notes de Botanique Mongo. Mémoires de la Classe des Sciences naturelles et médicales, (N.S.) ARSOM. T. XV, f. 3: 212 (in- $8^{\circ}$ ).

Ilumbe BG, Van Damme P, Lukoki LF, Joiris V, Visser M, Lejoly J. 2014. Contribution à l'étude des plantes médicinales dans le traitement des hémorroïdes par les pygmées Twa et leur voisin Oto de Bikoro, en RDC. Congo Sciences, 2(1) : 46-54.

Ilumbe BG. 2006. Usage des plantes chez les peuples de Bobangi. Mémoire de DEA, Université Libre de Bruxelles, 72 p.

Ilumbe BG. 2010. Utilisation des plantes en médecine traditionnelle par les Pygmées (Ba-twa) et les Bantous (Ba-Oto) du territoire de Bikoro, province de l'Equateur en RD Congo. Thèse Doct. Univ. Libre de Bruxelles, 237 p.

Kalanda K, Ataholo M, Ilumbe BG. 1995. Contribution à la connaissance des plantes médicinales du Haut-Zaïre: Plantes antihémorroïdaires de Kisangani. Rev.Méd. Pharm. Afr., 9(1): 51-58.

Kerharo J, Adam JG. 1974. Pharmacopée Sénégalaise Traditionnelle: Plantes Médicinales et Toxiques. Vigot Frères: Paris, $1011 \mathrm{p}$.

Kibungu KAO. 2004. Quelques plantes médicinales du Bas-Congo et leurs usages. DFID, London, United Kingdom. 197 pp.

Kluytmans J, Van Belkum A, Verbrugh H. 1997. Nasal carriage of Staphylococcus aureus: epidemiology, underlying mechanisms, and associated risks. Clin. Microbiol Rev., 10(3):505-20. DOI: 10.1128/CMR.10.3.505.
Latham P. 2004. Useful plants of Bas-Congo province, Democratic Republic of the Congo. DFID, London, United Kingdom, $320 \mathrm{p}$.

Lebrun J, Stork A. 2006. Tropical African Flowering Plants, Ecology and Distribution, vol 2, EuphorbiaceaeDicapetalaceae. Conservatoire et Jardin Botanique de la Ville de Genève, 306p.

Lebrun JP, Stork AL. 1991-1997. Énumération des Plantes à Fleurs d'Afrique Tropicale. Publ. H.s. Conservatoire Jardin Botanique Genève 7(1-4).

Legendre P, Legendre L. 1998. Numerical Ecology. Developments in Environnemental Modelling, 20. Elsevier Sciences B.V: Amsterdam; 853 p.

Marlier G. 1958. Recherches Hydrologiques au Lac Tumba. Den Haag : Uitgeverij Dr. W. Junk, 352-385.

Martin GJ, 1995. Ethnobotany: A Methods Manual. Chapman and Hall: London, UK.

Miller M, Cook HA, Furuya EY, Bhat M, Lee MH, Vavagiakis $\quad P$, Visintainer P, Vasquez G, Larson E, Lowy FD. 2009. Staphylococcus aureus in the community: colonization versus infection. PLoS ONE, 4(8):e6708.

Milliken W. 1997. Traditional anti-malarial medicine in Roraima, Brazil. Economic.

Milliken W, Miller RP, Pollard SR, Wandelli EV. 1992. Ethnobotany of Waimiri Atroari Indians of Brasil. Royal Botanic Gardens, Kew, London, UK, 146p.

Nana-Sanon P. 2005. Plantes médicinales utilisées pour les soins des enfants dans la commune de Ouagadougou: inventaire et culture de quelques espèces. Mémoire de fin d'études d'Ingénieur des Eaux et Forêts, $75 p$. 
O.M.S. 1993. Classification statistique internationale des maladies et des problèmes de santé connexes. OMS, 10ème révision vol.1, Genève, 1335 p.

O.U.A. 1985. Pharmacopée africaine. 1. CSTR/OUA, Lagos, Nigeria, $274 \mathrm{p}$.

Olivier M, Sanou L. 2003. Contribution à l'étude des plantes médicinales des jachères de l'ouest du Burkina Faso. Ethnopharmacologia, 30: 47-59.

Philippe PPB, Bénédicte RDL-V, Nathalie PDD, Selim APA, Samir PDB, Delphine AMA et al. 2004. Prescription des antibiotiques par voie locale dans les infections cutanées bactériennes primitives et secondaires. Médecine thérapeutique, 10(5): 365-368.

Phillips OL, Gentry AH, Reynel C, Wilkin P, Ga'lvez-Durand BC. 1994. Quantitative ethnobotany and Amazonian conservation. Conservation Biology, 8: 225-248.

Rawat AKS, Mehrotra S, Tripathi SC, Shome U. 1997. Hepatoprotective activity of Boerhaavia diffusa L. roots - a popular Indian ethnomedicine. Journal of Ethnopharmacology, 56: 61-66. DOI: https://doi.org/10.1016/S03788741(96)01507-3

Safdar N, Bradley EA. 2008. The risk of infection after nasal colonization with Staphylococcus aureus. Am. J. Med., 121(4): 310-315.

Satheesh MA and Pari L. 2004. Antioxidant effect of Boerhavia diffusa L. in tissues of alloxan-induced diabetic rats. Indian Journal of Experimental Biology, 42(10): 989-992.

Nene Bi SA, Traore F, Soro TY, Souza A. 2009. Etudes phytochimique et pharmacologique de Bridelia ferruginea benth (euphorbiaceae) sur la motricité du
Taenia coli de cobaye. Afrique Science, 05(2): 305- 320.

Slamet S, Budi R. 2001. Boerhavia L. In Plant Resources of South-East Asia No 12(2): Medicinal and poisonous plants 2, van Valkenburg JLCH, Bunyapraphatsara $\mathrm{N}$ (Ed). Backhuys Publishers: Leiden, Netherlands; 111-

Stokes ME, Davis CS, Koch GG. 2000. Categorical Data Analysis Using the SAS System (2nd ed). NC, SAS Institute Inc. 18: USA.

Stevens DL, Bisno AL, Chamber HF, Patchen Dellinger E, Goldstein EJC, Gorbach SL, Hirschmann JV, Kaplan SL, Montoya JG, Wade JC. 2014. Practice Guidelines for the Diagnosis and Management of Skin and Soft Tissue Infections. Infectious Diseases Society of America. Clinical Infectious Diseases, 59(2): $\quad 10-52 . \quad$ DOI: https://doi.org/10.1086/497143.

Talan DA, Mower WR, Anusha K, Abrahamian FM, Lovecchio F, Karras DJ, Steele MT, Rothman RE, Hoagland R, Moran GJ. 2016. TrimethoprimSulfamethoxazole versus Placebo for Uncomplicated Skin Abscess. $N$ Engl J Med., 374: 823-832. DOI: 10.1056/NEJMoa1507476

Terashima H, Ichikawa M. 2003. A comparative ethnobotany of the Mbuti and Efe hunter-gatherers in the Ituri forest, Democratic Republic of Congo. African Study Monographs, 24(1-2): 1168. DOI : $10.14989 / 68220$

Thomas E, Vandebroek I, Sanca S, Van Damme P. 2009. Cultural significance of medicinal plant families and species among Quechua farmers in Apillapampa, Bolivia. Journal of Ethnopharmacology, 122: 60-67. DOI: https://doi.org/10.1016/j.jep.2008.11.021 
Trochain. 1980. Ecologie végétale de la zone intertropicale non-désertique. Université Paul Sabatier, Toulouse, 468 p.

Trotter RT, Logan MH. 1986. Informant consensus: a new approach for identifying potentially effective medicinal plants. In: Etkin NL. (Ed.), Plants in Indigenous Medicine and Diet. Redgrave Publishing Company: Bedford Hill, New York; 91-112.

Trouiller P, Rey JL. 1997. Maladies dominantes de la zone intertropicale, recherches et développement pharmaceutique: les médicaments indigènes. In ReMed., 16: 9-10.

Van Dijk JFW. 1999. Non-timber forest products in the Bipindi-Akom II region, Cameroon. A socio-economic and ecological assessment. The TropenbosCameroon programme, $197 \mathrm{p}$.

Voeks RA. 2004. Disturbance pharmacopoeias: medicine and myth from the humid tropics. Annals of the Association of American Geographers, 94(4): 868-888.

White F. 1983. The vegetation map of Africa. A descriptive memoir. UNESCO, Natural Ressources Research, 20: 1356.

Zerbo P, Millogo-Rasodimby J, NacoulmaOuedraogo OG, Van Damme P. 2007. Contribution à la connaissance des plantes médicinales utilisées dans les soins infantiles en pays San, au Burkina Faso. Int. J. Biol. Chem. Sci., 1(3): 262274. 